\title{
A Self Driving Car using Machine Learning and IOT
}

\author{
${ }^{1}$ Prof. Omprakash Yadav, ${ }^{2}$ Aman Sharma, ${ }^{2}$ Dion Philip, ${ }^{2}$ Boris Alexander \\ ${ }^{1}$ Professor, Student, ${ }^{2}$ Department of Computer Engineering, \\ Xavier Institute of Engineering, Mumbai-400016, India \\ amansharma44007@gmail.com, Omprakash.y@xavier.ac.in, dionphilip28@gmail.com, boriloui7@gmail.com
}

\begin{abstract}
This paper aims to represent a mini version of self-driving automotive victimization IOT with raspberry pi and Arduino UNO working as a main processor chip, the $8 \mathrm{mp}$ high resolution pi camera can offer the specified data and thus the raspberry pi can analyze the data(samples) and it will get trained in pi with neural network and machine learning algorithm which could finish in detection road lanes, traffic lights and thus the automotive can alternate consequently. to boot to these options the automotive can overtake with correct LED indications if it comes across associate obstacle.
\end{abstract}

\section{INTRODUCTION}

Automated vehicles area unit technological development in the field of cars. tho' the machine-controlled vehicles sq.measure for ease of man but they are the foremost high priced vehicles. In the paper considering the varied choices and also the worth, on a small scale a three wheel conveyance Robotic epitome has been designed which is able to automatically reach the destination of another vehicle thereto it's alleged to follow. We have targeted on a pair of applications of AN Automated Vehicles here and designed a epitome vehicle for that. The one major issue is throughout significant vital a driver has to endlessly push brake, accelerator and clutch to move to destination slowly. we've got projected a solution to relax the propulsion in this state of affairs by making vehicle sensible enough to build selections mechanically and move by maintaining a specified distance from vehicles and obstacles around. The second issue is once a pair of vehicles have the same destination but one in every of the drivers doesn't acknowledge its route. The driver can build his vehicle follow the front vehicle if they are known and share their location to achieve identical destination. A machine Mobile automaton is utilized for analysis is given. The Mobile automaton consists of multiple sensors, that helps it to communicate with Google Maps API(Application Program Interface) and makes it make sure obstacles so on follow the route and move smoothly. The Mobile automaton connects directly to Google Maps API exploitation GPRS Module, gets route and moves in this direction. Whereas the supersonic sensors, which square measure used for epitome vogue, helps to avoid obstacles on run time. The traffic state of affairs in Asian nation ends up fashionable this project prototype, that aims at relaxing driver and creating an automated vehicle whose destination is dynamic in distinction to Google car, whose destination is static and fixed. This analysis has been a demand for Asian nation if enforced in real time. The remaining paper is organized as a result of the Section II shows the connected work done in done in. Later we have got discussed our methodology to implement this idea and solve the matter in Section III. Then we have got shown performance analysis of our Mobile automaton in Section IV and finally, we have got concluded the paper in Section V.

\section{LITERATURE SURVEY}

1. The paper "Working model of Self-driving automotive victimisation Convolutional Neural Network, Raspberry Pi and Arduino" by Hindu deity Kumar religious belief. The planned model takes a picture with the assistance of Pi cam attached with Raspberry Pi on the automotive. The Raspberry Pi and also the laptop computer is connected to the same network, the Raspberry Pi sends the captured image to the Convolutional Neural Network. The image is gray-scaled before passing it to the Neural Network. Upon prediction the model provides one amongst the four output i.e. left, right, forward or stop. When the result's expected corresponding Arduino signal is triggered that successively helps the automotive to maneuver in a very particular direction with the assistance of its controller.

2. The paper "Self-driving automotive ISEAUTO for analysis and education" conferred by Raivo Sell, Anton Rassõlkin, Mairo Leier, Juhan-PeepErnits, describes an ISEAUTO project, the primary selfdriving automotive project in Esthonia is enforced at Tallinn University of Technology in cooperation with associate degree Estonian automotive company. ISEAUTO works in analysis and educational project 
targeted on the planning and development of a machine-controlled vehicle in cooperating with a personal company and students.

3. The paper conferred by T. Banerjee, S. Bose, A. Chakraborty, T. Samadder Bhaskar Kumar, T.K. Rana "Self Driving Cars: A Peep into the Future".

This paper elaborates a few distinctive technique embedded controller style of a self-driving, electrified, accident proof and GSM destination guided vehicle. A GPS module accurately tracks the location of the automotive, supply and destination, and mapping the co-ordinates provides navigation Speeds of the vehicle is mechanically controlled by keeping a secure distance, that may be a perform of velocity, having the vehicle before read. Distance of the front and facet vehicle square measure endlessly monitored by a stepper motor controlled rotating distance activity detector and also the ordinance as well as track dynamical square measure done consequently. It conjointly prevents collision because of associate degree obstacle.8-megapixel pi-camera with image processing unit has been used to sense traffic light and traffic density on road.

4. The paper "Self-Driving and Driver restful Vehicle", bestowed by Qudsia Memon, Muzamil Ahmed, Shahzeb Ali, Azam Rafique Memon, Wajiha Shah during this paper they need designed 2 applications of Associate in Nursing autonomous vehicle, which can help the driving force to relax for the restricted length of time. It conjointly presents a thought that focuses on modified conception of Google automobile, the Google automobile has to reach the static destination automatically; during this prototype, they created the dynamic destination. Here self-driving automobile can follows a vehicle that is moving on an explicit route. This model can follow that vehicle.

5. The paper "The problems and the Possible Solutions for Implementing Self-Driving Cars in Bangladesh" presented by prophet male monarch Bin Ahmed, Md. Saef Ullah Miah, Md. Muneef Anjum Timu, Shakura Akter, Md. Sarker.b. a number of the problems of Bangladeshi roads square measure highlighted in an exceedingly paper published Organization in 2004. Google automobile among other things, will calculate the foremost economical path, abide native traffic rules, park once necessary and change lane if needed.

6. The paper "Real-time multiple vehicle detection and tracking from a moving vehicle", by Margrit Betke, Esin Haritaoglu, Larry S. Davis. they need enclosed modules for detection of alternative vehicles on the road. The NavLab project at Carnegie Andrew W.
Mellon University uses the chop-chop Adapting Lateral Position Handler to determine the coordinates of the road ahead and the appropriate steering direction. RALPH automatically steered a Navlab vehicle $98 \%$ of a visit from Washington DC to city, California, a distance that is of over 2800 miles. They have added a module for automobile following. A module is employed for police work reordering vehicles, and a trinocular stereo module (three read vision) for police work distant obstacles were other to reinforce and improvise the Navlab performance of car.

7. The paper "Driver help System for Lane Detection and Vehicle Recognition with Night Vision", by ChunChe Wang, Shih-Shinh Huang and Li-Chen Fu, PeiYung Hsiao. It aims to boost driving, by making Associate in Nursing help system. To enhance driver's safety in the dead of night time the formula includes lane detection along side vehicle recognition system. It will find Lane that helps to localize the markers. Operation like cagy edge extraction is finished to extract edge map to that matching technique is applied followed by the selection of potentials edge points. Finally linking is done to localize the lane lines.

8. The paper "Traffic light-weight Detection and Recognition for Self Driving Cars exploitation Deep Learning”, by Ruturaj Kulkarni, Shruti Dhavalikar, Sonal Bangar. There square measure many object detection architectures available like Single Shot Multibox Detector (SSD), Faster Region primarily based Convolutional Neural Networks (R-CNN), Region primarily based totally (R-FCN) that incorporates feature extractors like ResNet-101, Inception-V2, Inception-V3, Mobile internet etc. The selection of design and have extractor is trade-off between speed and accuracy that your application wants For the stoplight detection considering the appliance demand and available process resources, quicker R-CNN Inception-V2 model is employed that serves the accuracy and speed trade-offs. The model is trained on the on top of mentioned dataset wherever loss is reported at every step of coaching. The model is trained on the NVIDIA GEFORCE 940M GPU exploitation TensorFlow.

9. The paper "Driverless Intelligent Vehicle for Future Public Transport supported GPS", by R.Mohanapriya, L.K.Hema, Dipesh warkumar Yadav, Vivek Kumar Verma. It involves arming GPS and GSM system on a four wheeled golem. The GPS system steers the robot and is capable of reaching from one purpose to another with none human intervention. While in the former 
one with the assistance of GSM system they promise to report felony just in case is there's any. An SMS alert is distributed to the vehicle owner coverage about the problem and as a results of it, the owner of the car will switch the ignition off and within the latter one the project states that vehicle will solely be turned on if the approved person sends a predefined location to the car.

10. The paper bestowed by Giuseppe Lugano "Virtual assistant and self-driving cars", introduced US The virtual assistant may be a specific code practicality originally planned among the "desktop" computing atmosphere to support the user within the learning and use of a selected code package (e.g. word processor, spreadsheet). the most purpose of virtual assistants was to extend the productivity and potency of the user with a selected product.

11. The paper bestowed by Dong, D., Li, X., \& Sun, “A Vision-based technique for up the protection of Selfdriving" offers careful read regarding however they developed a machine that is in a position to find traffic signs and lanes and road segmentation.

12. The paper bestowed by Straub, J., Amer, W., Ames, C., Dayananda, K. R, A.Jones, Miryala, Shipman “An Internetworked Self-Driving automobile System-of Systems", projected Associate in Nursing economical approach of creating communications between 2 or a lot of cars in an exceedingly particular system to stay the traffic less full.

\section{METHODOLOGY}

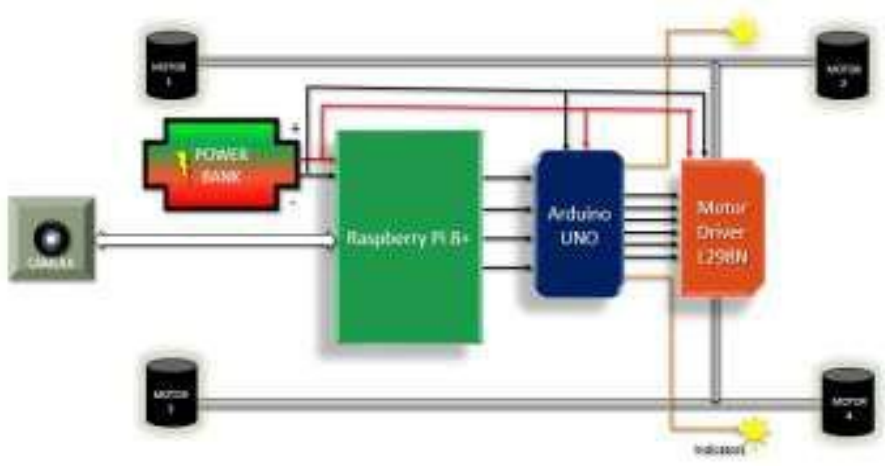

3.1 Raspberry pi camera:

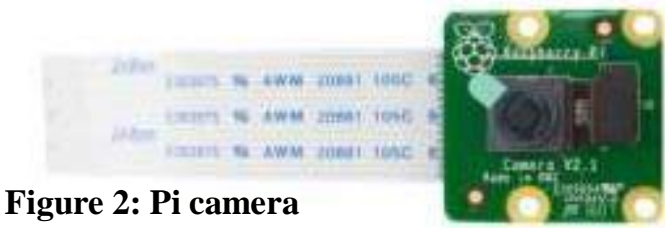

The pi-camera we have a tendency to square measure mistreatment is V2 version that comes with specifications like $8 \mathrm{mp}$ camera and supports upto $1080 \mathrm{p} 30$ resolutions beside IMX219 device and F2.9 aperture. This pi cam captures the pictures of surroundings and send them to the raspberry pi for more process.

\subsection{Raspberry pi:}

The raspberry pi is that the main processor here. Popularly called low value single board pc. we have a tendency to square measure mistreatment raspberry pi $3 \mathrm{~B}+$ version for image process. With the assistance of Open $\mathrm{CV}$ code, a machine learning algorithmic rule is enforced and also the pictures square measure trained in numerous lighting conditions mistreatment neural network technology. more the choices taken by the raspberry pi square measure sent as commands to Arduino.

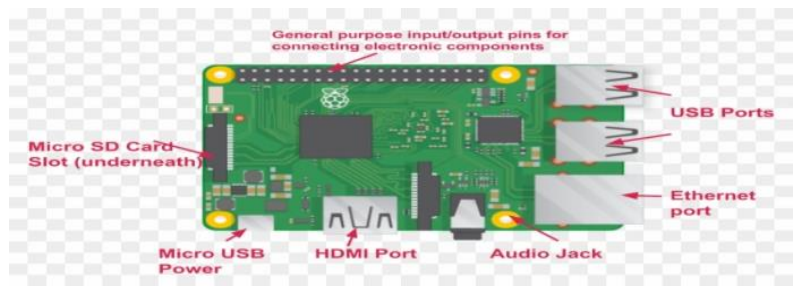

\section{Fig.3 Raspberry pi Board}

\subsection{Arduino UNO:}

Arduino Uno is ATmega329P primarily based microcontroller board. it's wide well-liked for mini comes. In our project Arduino is I accustomed management forward, backward, left and right movement of car. All the functions square measure preprogrammed in Arduino mistreatment Arduino IDE. once obtaining command from pi, the Arduino can send signal to motor driver circuit to require the suitable movement tutored by Arduino.

\subsection{L298N Motor Driver:}

It is a basic motor driver module accustomed drive dc motors similarly as stepper motors too. $\mathrm{H}$ bridge is employed beside L298 IC to drive motors. H bridge could be a circuit that may drive current in polarity and can be controlled by pulse breadth modulation (PWM)

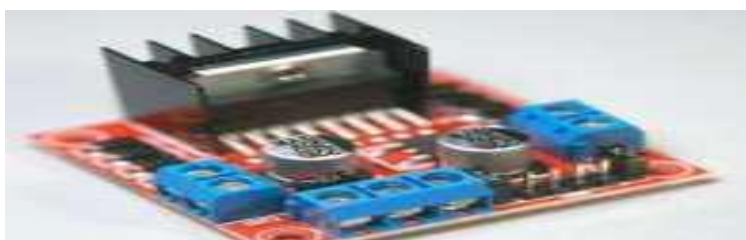

Figure 4: L298N Motor Driver: 


\subsection{LED indicators:}

LED indicators square measure placed at the rear of chassis. whereas taking $\mathrm{U}$ flip or if it detects Associate in Nursing obstacle indicator can have lit up to allow correct indications. Its programming are drop in IC 74LS164 and can be controlled through Arduino command.

\section{FLOWCHART}

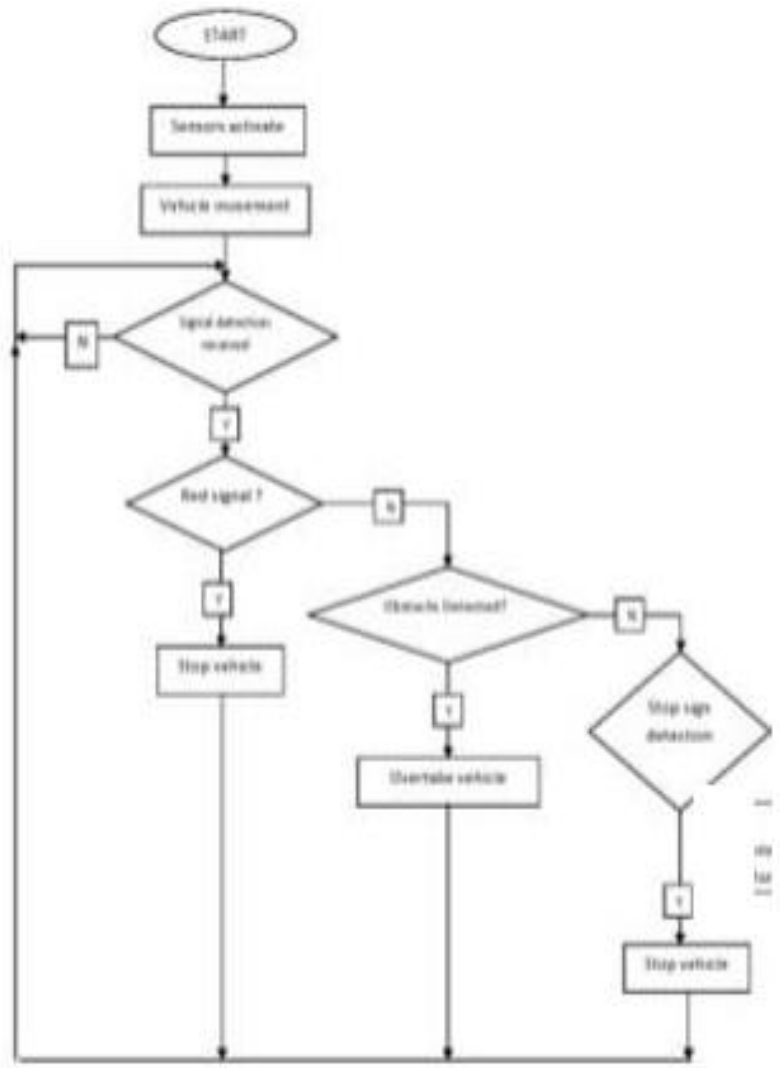

As the vehicle starts all the sensors and hardware components gets activated camera is capturing pictures raspberry pi begins operating and automobile can start moving. While processor starts process the pictures it'll hunt for three things.

1. red traffic light

2. obstacle

3. stop sign

If anyone of this detected then raspberry pi sends appropriate signal to Arduino to figure additional. If red signal is detected raspberry pi offers command to Arduino Uno to stop the automobile till red is became inexperienced. If stop sign is detected, then automobile can stop for specific planned time limit if associate degree obstacle is detected then vehicle can stop and overtake it by giving correct turning indications.

\section{RESULTS:}

Stop sign detection:
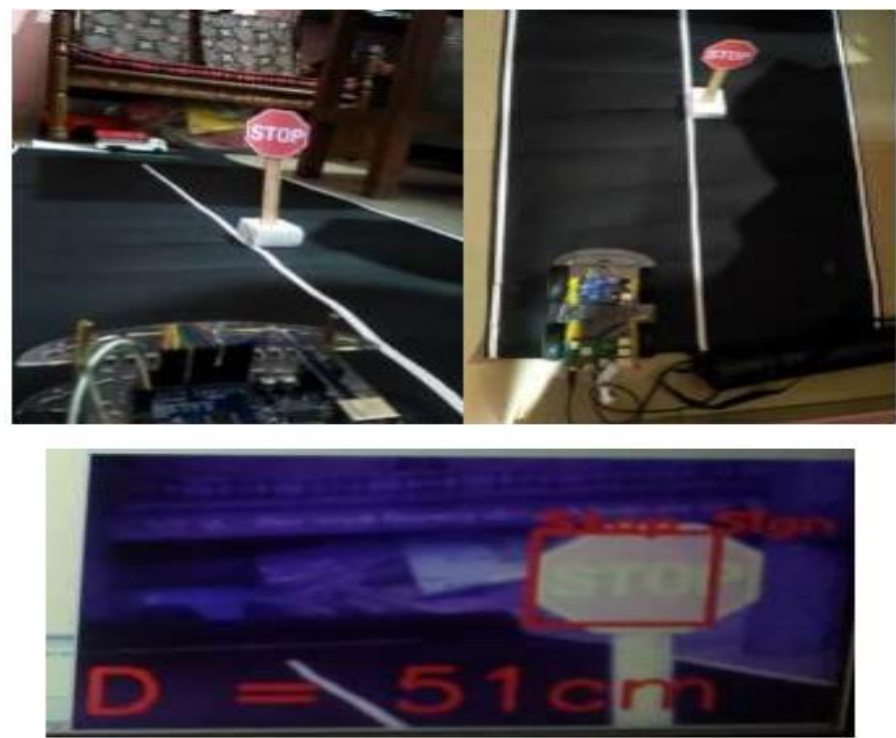

Lane detection

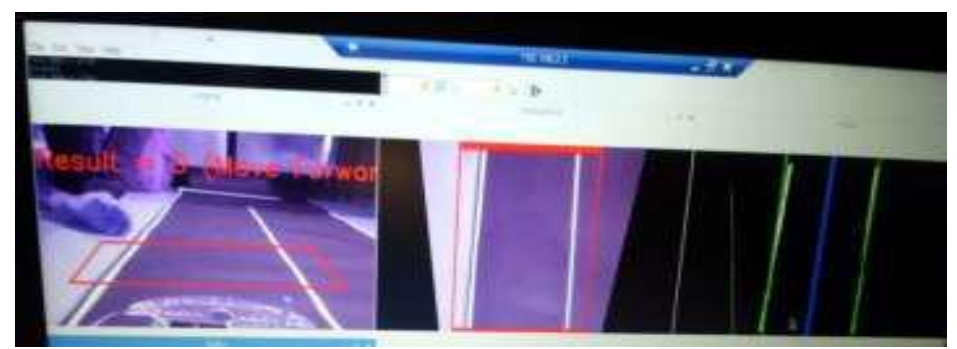

Obstacle detection

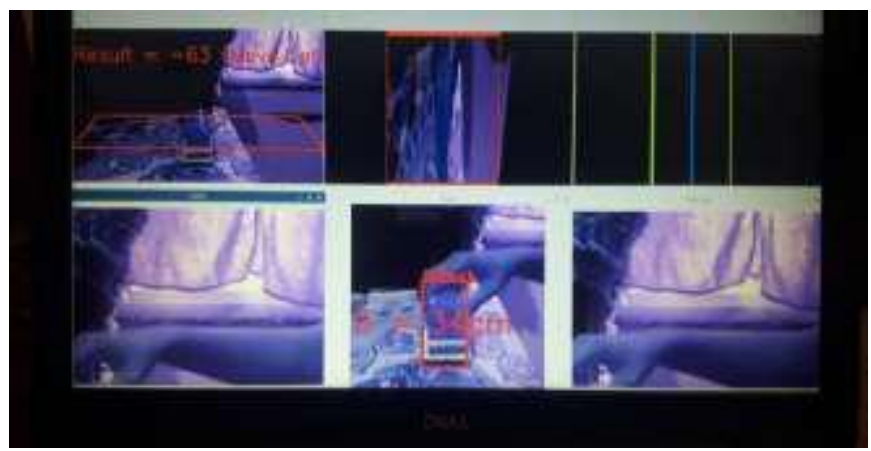


Traffic Light detection

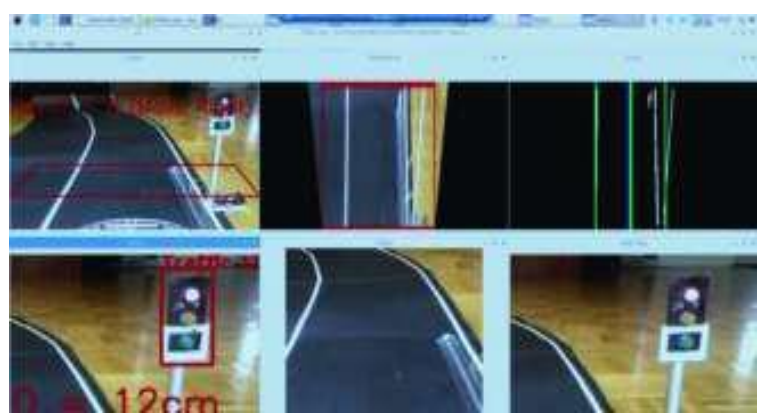

\section{CONCLUSION}

The planned self-driving automotive is with success created, enforced and tested. The vehicle is trained with quite two hundred samples of pictures in numerous lighting conditions. In varied testing done by U.S.A. it's found that despite of victimisation high resolution V2 version of Raspbian camera lighting and totally different environmental conditions could have an effect on the choice taken by the vehicle to beat this minor issue the coaching ought to be done exactly with excellent frame rates. This paper clearly describes the operating methodology of our autonomous vehicle.

\section{FUTURE SCOPE}

There is large scope of self-driving cars in future, the assorted automobile firms area unit rising their autonomous cars apace creating them a lot of correct and secured. By victimisation multiple cameras and sensors, the accuracy is improved. planning a system wherever each automotive is interconnected to near cars can avoid hold up in future.

\section{REFERENCES}

[1] Aditya Kumar Jain, "Working model of Self-driving car using Convolutional Neural Network, Raspberry $\mathrm{Pi}$ and Arduino", Proceeding of the 2nd International Conference on Electronics, Communication and Aerospace Technology (ICECA), IEEE, 2018, pp: 1630- 1635.

[2] Raivo Sell, Anton Rassõlkin, Mairo Leier, Juhan-Peep Ernits, "Self-driving car ISEAUTO for research and education"in 19th international conference on Research and Education in Mechatronics (REM), IEEE, 2018, pp: 111-116.

[3] T. Banerjee, S. Bose, A. Chakraborty, T. Samadder, Bhaskar Kumar, T.K. Rana, "Self Driving Cars: A Peep into the Future", IEEE, 2017, pp: 33-38.

[4] Qudsia Memon, Muzamil Ahmed and ShahzebAli, "Self Driving and Driver Relaxing Vehicle", in IEEE 2016, pp: 170174.

[5] Mohammad Faisal Bin Ahmed, Md. Saef Ullah Miah, Md. Muneef Anjum Timu, Shakura Akter, Md. Sarker.b, "The
Issues and the Possible Solutions for Implementing Self Driving Cars in Bangladesh" in Regin 10 Humanitarian Technology Conference(R10-HTC), IEEE,2017, pp: 250-254. 